\title{
Disformal transformations and the motion of a particle in semi-classical gravity
}

\author{
Sandip Chowdhury ${ }^{\mathrm{a}}$, Kunal Pal ${ }^{\mathrm{b}}$, Kuntal Pal ${ }^{\mathrm{c}}$, Tapobrata Sarkar ${ }^{\mathrm{d}}$ \\ Department of Physics, Indian Institute of Technology, Kanpur 208016, India
}

Received: 5 March 2021 / Accepted: 14 October 2021 / Published online: 25 October 2021

(C) The Author(s) 2021

\begin{abstract}
The approach to incorporate quantum effects in gravity by replacing free particle geodesics with Bohmian non-geodesic trajectories has an equivalent description in terms of a conformally related geometry, where the motion is force free, with the quantum effects inside the conformal factor, i.e., in the geometry itself. For more general disformal transformations relating gravitational and physical geometries, we show how to establish this equivalence by taking the quantum effects inside the disformal degrees of freedom. We also show how one can solve the usual problems associated with the conformal version, namely the wrong continuity equation, indefiniteness of the quantum mass, and wrong description of massless particles in the singularity resolution argument, by using appropriate disformal transformations.
\end{abstract}

\section{Introduction}

More than 25 years ago, Bekenstein [1] showed that in theories of gravity where two distinct geometries are present, they are, in general, related by a disformal transformation, which is a generalization of the conformal transformation. In such situations, the gravitational dynamics is controlled by the metric and is called gravitational geometry, whereas matter dynamics takes place on a geometry that is disformally related to the metric, and called physical geometry. This is a notable departure from general relativity (GR), where the dynamics of both gravity and matter are determined by the metric, but is common in scalar-tensor theories of gravity, such as Brans-Dicke theory, in which the two geometries are related by a conformal transformation. The purpose of this paper is to establish the nature of disformal transformations in the context of Bohmian mechanics in gravitational

\footnotetext{
a e-mail: sandipc@iitk.ac.in

b e-mail: kunalpal@iitk.ac.in

c e-mail:kuntal@iitk.ac.in

d e-mail: tapo@iitk.ac.in (corresponding author)
}

backgrounds, and to show that it solves a few important problems that arise in an usual treatment popular in the literature, that uses conformal transformations instead.

Bohmian mechanics [2-4] is an important tool in a semiclassical understanding of the full theory of quantum gravity [5]. Formulating such a quantum theory of gravity is of course a formidable challenge, with the metric playing the role of a quantum operator, and with quantization conditions on space and time. The somewhat simpler semi-classical approach, in which the metric is classical, has been popular over decades. In Bohmian mechanics, the particle trajectories are determined by suitable wavefunctions, and the statistical distribution of particle positions is given by the modulus squared of this wavefunction. In this first quantized approach, one replaces the geodesic motion of freely falling particles in a curved space-time by corresponding Bohmian trajectories. In such situations, geodesic equations are typically modified by an additional force term coming from a quantum potential [6-9]. This kind of reasoning has been used to deal with the usual singularity problem of classical general relativity [6].

Importantly, it is known that there is a close relationship between conformal transformations and the motion of a particle in Bohmian quantum mechanics. Namely, if we consider the quantum motion of a particle in a flat background, then this is equivalent to classical motion (one where the effect of quantum potential is absent) on a curved background which is related to the previous one by a conformal transformation. The conformal factor is a function of the modulus of the wavefunction, and is hence related to the quantum potential (see for e.g. [9-11,13] and references therein).

To understand this equivalence beyond the existing literature, in the first part of this work, we consider a Klein-Gordon type field, which is governed by Bohmian mechanics, and is non-minimally coupled to gravity. After deriving the Raychaudhuri equation, we discuss how we can transform to a conformally related frame (with the conformal factor being a variable particle mass) where the motion of the particle 
is force free, but the corresponding field equations have the information of the quantum nature of the particle. We argue how to deal with the singularity problem in this frame where the quantum force is absent by working out a particular example of such transformation with a suitable choice of the wave function, in a Schwarzschild background.

In this context, an important question to ask is the following. If the physical geometry on which the particle moves is different from the gravitational geometry, can we perform an analysis similar to the one described above, where the particle, moving in the physical geometry, is a quantum mechanical particle obeying Bohmian mechanics ? Is it possible to incorporate quantum effects in the disformal factor as in the conformal case? We show it is possible, by deriving the relation between the acceleration equations and then relating the factors of the transformation with quantum potential.

Next, in a minimally coupled scenario, we show that by using the equivalent description in the disformal frame, we can solve some well known problems that are present in the conformal version of theory described before. For example, in presence of the quantum potential, the mass of a particle is modified to a variable one (known as the quantum mass of the particle), and this is not always positive definite, so that the theory may have tachyonic modes [3]. If we transform to a conformal frame where the quantum potential is absent, the particle mass becomes constant, but the transformation itself is not definite for such imaginary quantum mass. Furthermore the continuity equation in the transformed frame does not have the desired form to define a suitable probability density $[14,15]$. One of the main contributions of this paper is to show that instead of transforming to a conformal frame, if we use a disformal transformation, all of the above mention problems can be solved. The modified geodesic equation and the continuity equation fix the transformation factors such that have positive definite values even for a particle with negative quantum mass.

In the last part this work we address a related question. If the force on the particle due to it's quantum nature is absent in the conformal frame, what happens for a massless particle moving in null trajectory in that frame. It is known that, by the nature of construction of conformal transformation the null geodesics remains invariant, they do not feel any force in any of the two conformally related frames. In particular what happens to the argument used to avoid the singularity problem. Namely, how can null trajectories not cross if they do not feel any quantum force? We shall show how to answer this question by using the fact that under a general disformal transformation, a null trajectory of one frame is not null in another frame $[1,16]$. By doing a disformal transformation in a direction different from the wave vector of the photon, we show that photon does not moves in a null trajectory in the gravitational geometry, and hence can avoid the singularity.
This paper is organized as follows. In Sect. 2, we briefly review the non-geodesic motion of the Bohmian particle in curved spacetime. Then we elaborate how the relation between the motion of this particle on a fixed background geometry can be equivalently described by a force free motion in a conformal frame, where the quantum effects are hidden in the energy momentum tensor. In Sect. 3, by assuming a minimal coupling between wavefunction and background geometry we describe how the previous set up may be generalized to disformally related spacetimes. Finally, in Sect. 4, we demonstrate how one can solve the usual problems that appear in a conformally transformed frame by using disformal transformations. The paper ends with conclusions in Sect. 5. Throughout this paper, we work in natural units and set $G=c=\hbar=1$.

\section{Bohmian motion on a classical background}

\subsection{The action and the Klein-Gordon equation}

We consider a spinless quantum particle of mass $m$ moving on a timelike path in a fixed classical background. The normalized wave function of such a particle $\Psi\left(x^{\mu}\right)$ can be written in terms of two single valued real functions $\mathcal{R}\left(x^{\mu}\right)$ and $\mathcal{S}\left(x^{\mu}\right)$, which are the modulus and the phase function respectively, as $\Psi\left(x^{\mu}\right)=\mathcal{R}\left(x^{\mu}\right) e^{i \mathcal{S}\left(x^{\mu}\right)}[3,4]$. Substituting this form of wave function in the Schrodinger equation, and separating the real and imaginary parts, one can show that, with the four-momentum associated with a particle of mass $m$ guided by this wave function given by $p_{\mu}=\partial_{\mu} \mathcal{S}\left(x^{\mu}\right)$, these two equations can be interpreted as a quantum Hamilton-Jacobi equation, and a continuity equation with probability density $\rho=\mathcal{R}^{2}[2,3]$, respectively. But the Schrodinger equation is not a manifestly covariant equation. Thus to write down the relativistic version of the quantum Hamilton-Jacobi equation and the continuity equation, a popular approach in the literature has been to instead consider a Klein-Gordon type equation of a particle (whose dynamics is governed by the Bohmian mechanics) described by the wavefunction $\Phi\left(x^{\mu}\right)$ which is assumed to be moving on a fixed classical curved background $\left(g_{\mu \nu}\right)$. Such models are considered previously in the literature in the context of Bohmian mechanics in [5$9,13]$, also see the books [11,12] for reviews of previous works on the usage of the Klein-Gordon equation in gravity. Indeed, as we have mentioned before, in relativistic quantum mechanics, different conceptual problems arise in the Bohmian interpretation of a single particle Klein-Gordon equation, e.g., indefiniteness of the quantum mass and the wrong continuity equation. Here we will show that such problems can be avoided by transforming to a disformally related frame. 
We will start with the following action, with a nonminimal coupling term between the particle wavefunction $\Phi$ and the Ricci scalar $R$ whose variation will give us the desired Klein-Gordon type equation :

$$
\begin{aligned}
S_{g r}+S_{\Phi}+S_{m}= & \int d^{4} x \sqrt{-g} F(|\Phi|) R \\
& +\int d^{4} x \sqrt{-g}\left[-\frac{1}{2} g_{\mu \nu}\left(\nabla^{\mu} \Phi^{*}\right)\left(\nabla^{\nu} \Phi\right)\right. \\
& \left.-\frac{1}{2} m^{2}|\Phi|^{2}\right]+\int d^{4} x \sqrt{-g} \mathcal{L}_{m}\left(g^{\mu \nu}, \lambda_{i}\right) .
\end{aligned}
$$

This action is commonly used popular alternatives to GR, called the scalar-tensor theories (the literature on the subject is vast, see, e.g., $[17,18])$. There, $\Phi$ is more appropriately a scalar field. Here on the other hand, $\Phi\left(x^{\mu}\right)$ (a scalar function) is the first quantized wavefunction of the particle. In this paper no second quantization is imposed upon $\Phi\left(x^{\mu}\right)$. In Eq. (1), $F(|\Phi|)$ is a function of the norm of the particle wavefunction and from now on we shall concentrate on a particular choice of $F(\Phi)$, namely $F(\Phi)=-\frac{1}{2} \epsilon|\Phi|^{2}$. The subscripts in the terms on the left hand side denote the actions of the gravitational part, that due to the particle wavefunction and the matter fields (collectively denoted as $\lambda^{i}$ ) respectively. The coupling between the Ricci scalar $R$, and the wavefunction $\Phi$ is assumed to be independent of whether it is in the quantum regime or can be approximately taken to be classical, i.e., independent of the energy scale of the system.

As before we can decompose the wavefunction in the polar form $\Phi=\mathcal{R}\left(x^{\mu}\right) e^{i \mathcal{S}\left(x^{\mu}\right)}$ with $\mathcal{R}\left(x^{\mu}\right)$ and $\mathcal{S}\left(x^{\mu}\right)$ being two single valued real function of spacetime coordinates. Now substituting this in the total action, with the above choice of the coupling function, we have

$$
\begin{aligned}
& S_{g r}+S_{\Phi}+S_{m} \\
& =\frac{1}{2} \int d^{4} x \sqrt{-g}\left[-\epsilon R \mathcal{R}^{2}-g_{\mu \nu}\left(\left(\nabla^{\mu} \mathcal{R}\right)\left(\nabla^{\nu} \mathcal{R}\right)\right.\right. \\
& \left.\left.\quad+\mathcal{R}^{2}\left(\nabla^{\mu} \mathcal{S}\right)\left(\nabla^{\nu} \mathcal{S}\right)\right)-m^{2}|\mathcal{R}|^{2}\right]+S_{m} .
\end{aligned}
$$

This from of the action manifestly provides the coupling between the particle wavefunction and the geometry. As can be directly seen, only the magnitude of the wavefunction couples with the Ricci scalar and also contributes to the potential. From Eq. (1), we see that gravitational dynamics is governed by the field equations $[17,19]$

$$
\begin{aligned}
G_{\mu \nu}= & -\frac{1}{\epsilon|\Phi|^{2}}\left(T_{\mu \nu}^{m}+T_{\mu \nu}^{\Phi}\right) \\
& +\frac{1}{|\Phi|^{2}}\left(\nabla_{\mu} \nabla_{\nu}|\Phi|^{2}-g_{\mu \nu} \square|\Phi|^{2}\right) \\
& \text { with } T_{\mu \nu}^{i}=-\frac{2}{\sqrt{-g}} \frac{\delta S_{i}}{\delta g_{\mu \nu}},
\end{aligned}
$$

where $i=m$, $\Phi$. Here, $G_{\mu \nu}$ is the Einstein tensor constructed from the metric $g_{\mu \nu}$, and $T_{\mu \nu}^{m}$ and $T_{\mu \nu}^{\Phi}$ are the energy momentum tensors associated with matter fields and $\Phi$ respectively (we do not write down the explicit expressions for these quantities, which are standard and can be found in the references cited above). On the other hand the particle wave function satisfy the following Klein-Gordon like equation

$\left[\square-m^{2}-\epsilon R\right] \Phi=0$.

In four spacetime dimensions, this equation is conformally invariant only when the mass term is set to zero and $\epsilon=\frac{1}{6}$ [20]. It is also important to notice that in [6], it was assumed that the background metric is non-dynamical in nature as a first approximation, i.e., backreaction was neglected. But in the general case with a non zero coupling between curvature and $\Phi$, this is not possible, unless of course we choose $\Phi$ to be not dynamical (this fact should be clear from the field Eq. (3) above).

Substituting the polar form of the wavefunction $\Phi=$ $\mathcal{R}\left(x^{\mu}\right) e^{i \mathcal{S}\left(x^{\mu}\right)}$ in the Klein-Gordon equation, and separating real and imaginary parts, or, alternatively directly using the second from of the action in Eq. (2) we get the following equations

$$
\begin{aligned}
\left(\nabla_{\mu} \mathcal{S}\right)\left(\nabla^{\mu} \mathcal{S}\right) & =-m^{2}-\epsilon R+\frac{\square \mathcal{R}}{\mathcal{R}} \text { and } \\
\nabla_{\mu} \mathcal{J}^{\mu} & =0 \text { with } \mathcal{J}_{\mu}=\mathcal{R}^{2}\left(\nabla_{\mu} \mathcal{S}\right) .
\end{aligned}
$$

The last term in the right hand side of the first relation above is known as the the quantum potential, which we shall denote as $f(\mathcal{R})$ i.e. with $\hbar^{2}$ momentarily restored: $f(\mathcal{R})=\frac{\hbar^{2} \square \mathcal{R}}{\mathcal{R}}$. After one defines the appropriate curved space generalization of the four-momentum of the particle i.e., $p_{\mu}=\nabla_{\mu} \mathcal{S}$, this equation gives the constraint on the magnitude of fourmomentum

$p_{\mu} p^{\mu}=-m^{2}-\epsilon R+\frac{\square \mathcal{R}}{\mathcal{R}}$.

On the other hand the second relation above represent a conservation equation for the current $\mathcal{J}^{\mu}$.

From the above Eq. (6), we glean that the norm of the fourmomentum is not constant, and given this magnitude of the four-momentum it will be important how one defines a fourvelocity vector from it. We do this by defining the normalized four-velocity for a timelike trajectory as $u^{\mu} u_{\mu}=-1$, so that [5]

$p^{\mu}=M(x) u^{\mu}, \quad M(x)=\sqrt{\left(m^{2}-f(\mathcal{R})+\epsilon R\right)}$.

With this definition, the four-velocity remains the same as in the classical trajectory but the particle's mass becomes a variable depending on the quantum amplitude $\mathcal{R}$, and this is 
known as the quantum mass of the particle. It is known that under an appropriate conformal transformation the action of a particle of variable mass becomes the action of a particle of constant mass (and vice versa). Using this fact in the next section we shall work in a conformally related frame, with conformal factor being appropriate function of quantum potential such that the dispersion relation of Eq. (6) transforms to the dispersion relation of a particle of constant mass.

On the other hand the second equation of Eq. (5) implies, with this interpretation of variable quantum mass, the conservation equation of the 4-current to be $\nabla_{\mu}\left(\mathcal{R}^{2} M u^{\mu}\right)=0$. Unfortunately however, now it is not possible to interpret this equation (as is done in the corresponding non-relativistic Bohmian treatment of Schrodinger equation) as a continuity equation with the probability density defined as $\rho=$ $\mathcal{R}^{2}$. Because the corresponding equation should look like $\nabla_{\mu}\left(\rho u^{\mu}\right)=0$ [15]. As mentioned above, one of the motivation to transform to conformal frame is to make the particle mass constant and hence the equation of motion a geodesics, but it is not possible (as we shall explain in Sect. 2.5 below), with the same transformation to make the conservation equation a continuity equation for probability density. Recently in [14] the authors have suggested a solution of this problem. In this work we shall propose an alternative one - use a disformal transformation rather than the conformal one.

As is clear from Eq. (6), due to presence of the quantum potential (the $f(\mathcal{R})$ term), the equation of motion of the particle is not a geodesic. Rather it contains an extra term coming form the force generated by the quantum potential. The straightforward way to find this force term in the acceleration equation is to take the directional derivative of the constraint relation of Eq. (6) by introducing a parameter (say $\tau$ ) along the particle trajectory (see [9] for a derivation along these lines). For later purposes however, we shall use a variational principle and write down the action along the particle trajectory between two points (say 1 and 2) as

$$
\begin{aligned}
& S\left[x^{\mu}(\tau), \eta(\tau)\right]=\int_{1}^{2} d \tau \mathcal{L}, \\
& \mathcal{L}=\frac{1}{2}\left[\eta^{-1} g_{\mu \nu} u^{\mu} u^{\nu}-\eta\left(m^{2}-f(\mathcal{R})+\epsilon R\right)\right],
\end{aligned}
$$

where $\eta$ is a Lagrange multiplier, and $u^{\mu}$ is the four-velocity of the particle, normalized as $u^{\mu} u_{\mu}=-1 .{ }^{1}$ Variation of the action with respect to $x^{\mu}$ gives the usual Euler-Lagrange equations, and a variation with respect to the Lagrange multiplier gives $\eta^{2}=-\frac{u^{\mu} u_{\mu}}{M^{2}}$, with $M$ being the quantum mass defined above. Now substituting this into the Lagrangian of Eq. (8), we have

$$
\mathcal{L}=\eta^{-1}\left[u^{\mu} u_{\mu}\right]=-M \sqrt{-g_{\mu \nu} u^{\mu} u^{\nu}},
$$

\footnotetext{
1 This four-velocity normalization is different from [6] but same as [9].
}

so that the corresponding four-momentum $p_{\mu}=\frac{\partial \mathcal{L}}{\partial \dot{x}^{\mu}}=$ $\frac{M}{\sqrt{-u^{\mu} u_{\mu}}} u_{\mu}$ satisfies the required constraint relation $p_{\mu} p^{\mu}=$ $-M^{2}$ (irrespective of the normalization of the 4-velocity). The acceleration equation corresponding to this Lagrangian is obtained by using the Euler-Lagrange equations, and is given by

$$
\begin{aligned}
u^{\mu} & \nabla_{\mu} u^{\nu} \\
& =-\frac{1}{2}\left(g^{\mu \nu}+u^{\mu} u^{\nu}\right) \nabla_{\mu} \ln \left(M^{2}\right) \\
& =-\frac{1}{2}\left(g^{\mu \nu}+u^{\mu} u^{\nu}\right) \nabla_{\mu} \ln \left[1-(f(\mathcal{R})-\epsilon R) / m^{2}\right] .
\end{aligned}
$$

As anticipated, the right hand side of Eq. (10) is non zero, and this indicates that the motion of a particle which is freely falling in the classical description is no longer force free for motion along a Bohmian trajectory. This term comes from the quantum potential. So unless $\mathcal{R}=$ constant, or it satisfies the equation $\square \mathcal{R}=0$, this term is non-zero. This matches with the acceleration equation derived in [9] from the momentum constrain relation (6), but it is different from the equation written in [6]. This is due to the different parameterization employed, namely, the term proportional to $u^{\mu} u^{\nu}$ can be absorbed by changing the parameter, although the first term can never be removed by any such redefinition of the parameter. Also note that the force term (from Eq. (10)) is perpendicular to the velocity vector, i.e., $u^{v} u^{\mu} \nabla_{\mu} u_{v}=u^{\mu} h_{\mu v}=0$, where we have defined the transverse metric $h_{\mu \nu}=g_{\mu \nu}+u_{\mu} u_{v}$.

Now consider a congruence of timelike particle trajectories with $u^{\mu}$ being the tangent to the trajectories. In a standard fashion [24], we consider $B_{\mu \nu}=\nabla_{\nu} u_{\mu}$ and calculate it's change along the particle trajectories as

$u^{\mu} \nabla_{\mu} B_{\alpha \beta}=\nabla_{\beta}\left(u^{\mu} \nabla_{\mu} u_{\alpha}\right)-B_{\alpha \mu} B_{\beta}^{\mu}-R_{\alpha \mu \beta \nu} u^{\mu} u^{\nu}$.

Taking the trace of this equation, and using the acceleration equation derived above, we arrive at the modified Raychaudhuri equation given by

$$
\begin{aligned}
\frac{d \theta}{d \tau}= & h^{\mu \nu}\left(\nabla_{\mu} \nabla_{\nu} L+\nabla_{\mu} L \nabla_{\nu} L\right)+\theta \frac{d L}{d \tau}-\frac{1}{3} \theta^{2}-\sigma^{\alpha \beta} \sigma_{\alpha \beta} \\
& +\omega^{\alpha \beta} \omega_{\alpha \beta}-R_{\alpha \beta} u^{\alpha} u^{\beta},
\end{aligned}
$$

where $a^{\alpha}=u^{\mu} \nabla_{\mu} u^{\alpha}$ is the acceleration vector, $\theta=B_{\mu}^{\mu}=$ $\nabla_{\mu} u^{\mu}$ is the expansion scalar, $\sigma_{\mu \nu}=B_{(\mu \nu)}-\frac{1}{3} \theta h_{\mu \nu}$ is the shear tensor and $\omega_{\mu \nu}=B_{[\mu \nu]}$ is the rotation tensor, and we have defined the function

$L=-\frac{1}{2} \ln \left[1-(f(\mathcal{R})-\epsilon R) / m^{2}\right]$.

The terms involving $L$ in the quantum Raychaudhuri equation are the contributions of the quantum effects, and the rela- 
tive contribution of these terms with respect to other classical terms determine whether the trajectory will reach a conjugate point or not [6]. Note that this form of Raychaudhuri equation is different from one given in [6], due to the different parameterization of the trajectory. It is also different from the one derived in [9], because those authors took $\nabla_{\alpha} h_{\mu \nu}=0$. We also record the expression of the deviation equation for the vector field $\xi^{\mu}$ between two non-geodesic curves as

$\frac{d^{2} \xi^{\mu}}{d \tau^{2}}=\xi^{\alpha}\left(u^{\mu} u^{\nu} \nabla_{\alpha} \nabla_{\nu} L+u^{(\mu} \nabla_{|\alpha|} u^{\nu)} \nabla_{\nu} L\right)+R_{\alpha \rho \nu}^{\mu} \xi^{\alpha} u^{\rho} u^{\nu}$

\subsection{Bohmian trajectories in a conformally related frame}

The purpose of this subsection is to discuss the transformation of the acceleration equation derived above, under a conformal transformation. If two metrics are related by a conformal transformation of the form $\tilde{g}_{\mu \nu}=\Omega^{2}(x) g_{\mu \nu}$, then it is well known that the relation between the acceleration equations in two frames are given by (see [19])

$\tilde{u^{\mu}} \tilde{\nabla}_{\mu} \tilde{u}^{v}=u^{\mu} \nabla_{\mu} u^{v}+h^{\mu v} \nabla_{\mu}(\ln \Omega)$.

From this relation, we see that if a particle moves along the geodesic of the transformed frame, then the motion in the frame $g_{\mu \nu}$ is non geodesic.

In our case, we choose to work in a frame that is related to the original metric by a conformal factor, such that the relation between the two metrics are ${ }^{2}$

$\tilde{g}_{\mu \nu}=\left(M^{2} / m^{2}\right) g_{\mu \nu}$.

We can conventionally express the above relations in terms of the action of a particle corresponding to the Lagrangian of Eq. (9), along the path $\gamma$ given by $x^{\mu}=x^{\mu}(\tau)$,

$$
\begin{aligned}
S_{m}\left[g_{\mu \nu}, \gamma, M\right] & =-\int_{\gamma} m \sqrt{1-\frac{f(\mathcal{R})-\epsilon R}{m^{2}}} \sqrt{-g_{\mu \nu} u^{\mu} u^{\nu}} d \tau, \\
u^{\mu} & =\frac{d x^{\mu}}{d \tau} .
\end{aligned}
$$

In the conformally transformed frame the corresponding action for a path $x^{\mu}=x^{\mu}(\tilde{\tau})$ is transformed into

$$
S_{m}\left[\tilde{g}_{\mu \nu}, \gamma\right]=-\int_{\gamma} \sqrt{-\tilde{g}_{\mu \nu} \tilde{u}^{\mu} \tilde{u}^{v}} d \tilde{\tau}, u^{\mu}=\frac{d x^{\mu}}{d \tilde{\tau}} .
$$

As can be readily seen by comparing them, the first form represents a particle having a variable mass $M$ defined in Eq. (7), and the second one is the action for a unit mass particle. If we

\footnotetext{
2 The conformal factor $\Omega^{2}$ is a dimensionless quantity. But in the following we shall often ignore the constant $\mathrm{m}^{2}$ factor for clarity.
}

write down the acceleration equations corresponding to these actions, they turn out just to be same equations derived before i.e., $u^{\mu} \nabla_{\mu} u^{\nu}+h^{\mu \nu} \nabla_{\mu}(\ln \Omega)$ and $\tilde{u}^{\mu} \tilde{\nabla}_{\mu} \tilde{u}^{v}=0$, and thus satisfy Eq. (15). The implication of this relation is straightforward. Namely, the motion in the conformal frame is free from the force of the quantum potential. If we denote the momentum corresponding to the conformal frame as $\tilde{p}^{\mu}$, then this can be shown to satisfy $\tilde{p}^{\mu} \tilde{p}_{\mu}=-1$.

If the motion in the conformal frame is force free, then where are the effects of the quantum potential in such a frame? The answer is simply that it is hidden inside the metric $\tilde{g}_{\mu \nu}$ via the conformal factor. In other words the quantum effects are in the field equations, so that the metric and also the energy momentum tensors are different. Indeed, starting from the action of Eq. (1) with $\epsilon=1 / 6$, making the transformation of Eq. (16), we obtain, after some algebra the following simplified action

$$
\begin{aligned}
S= & \frac{1}{2} \int d^{4} x \sqrt{-\tilde{g}}\left[-\frac{1}{6} \tilde{R}|\tilde{\Phi}|^{2}-\tilde{\nabla}_{\mu} \tilde{\Phi}^{*} \tilde{\nabla}^{\mu} \tilde{\Phi}-M^{-2}|\tilde{\Phi}|^{2}\right] \\
& +\int d^{4} x \sqrt{-\tilde{g}} \tilde{\mathcal{L}}_{m}\left(M^{-2} \tilde{g}_{\mu \nu}, \lambda_{(i)}\right),
\end{aligned}
$$

where we have redefined $\tilde{\Phi}=(\mathrm{M} / \mathrm{m})^{-1} \Phi$. Since the conformal factor $M^{2}$ is a real (or purely imaginary) number, $\tilde{\Phi}$ and $\Phi$ have different norms $(\mathcal{R})$, but they correspond to the same four-momentum $p_{\mu}=\nabla_{\mu} \mathcal{S}$.

The standard variation of this action with respect to the modified metric $\tilde{g}_{\mu \nu}$ and the modified wavefunction $\tilde{\Phi}$ gives the conformal version of Einstein equations and the equation of motion for the modified wavefunction (which are standard, see, e.g., [17] and [20]). The energy momentum tensors in the two frames are related by $\tilde{T}_{\mu \nu}^{m}=M^{-2} T_{\mu \nu}^{m}$ and hence transformed frame the EM tensor is no longer conserved, unless the trace of the EM tensor $\tilde{T}_{m}$ in the conformal frame vanishes, i.e., $\tilde{\nabla}_{v} \tilde{T}_{m}^{\mu \nu}=-\tilde{T}_{m}\left(\nabla^{\mu} \ln M\right)$.

\subsection{Conserved quantities}

If one wants to study the observational aspect of the problem of a particle moving along a quantum trajectory (Eq. (10)), in presence of a massive gravitational object (say a black hole), it is important to find out the conserved quantities. Because the motion is not that of a freely falling particle, it is not obvious if usual conserved quantities for stationary spacetime, namely energy and angular momentum are also conserved along Bohmian trajectories.

When a classical particle of mass $m$ moves in a geodesic satisfying $p^{\mu} \nabla_{\mu} p_{v}=0$ (here $p^{\mu}=m u^{\mu}$ ), the quantity $K_{v} p^{v}$ is conserved along the motion of the particle, i.e., $p^{\mu} \nabla_{\mu}\left(K_{\nu} p^{\nu}\right)=0$, where $K^{\mu}$ is a Killing vector which satisfies the Killing equation $\nabla_{(\mu} K_{v)}=0$. To find out how this equation is modified when the motion is along 
a quantum Bohmian trajectory, we take directional derivative of the constraint relation $p_{\mu} p^{\mu}=-M^{2}$ to obtain $p^{\mu} \nabla_{\mu} p_{v}=-M \nabla_{\nu} M(x)$. Now along quantum trajectory, we find

$$
\begin{aligned}
p^{\mu} \nabla_{\mu}\left(K_{\nu} p^{\nu}\right) & =p^{\mu} K_{\nu} \nabla_{\mu}\left(p^{\nu}\right)+p^{\mu} p^{\nu} \nabla_{\mu} K_{\nu} \\
& =-K^{\nu}\left[M \nabla_{\nu} M(x)\right]+p^{\mu} p^{\nu} \nabla_{(\mu} K_{v)} .
\end{aligned}
$$

We see that the Killing equation no longer implies that $K_{v} p^{v}$ is a conserved quantity. However, it can be checked that $p_{\mu} K^{\mu}$ is a conserved quantity, if $K^{\mu}$ is a conformal Killing vector associated with the metric $\tilde{g}_{\mu \nu}$, i.e., it satisfies the equation $\nabla_{(\mu} K_{v)}=-g_{\mu \nu} K^{\lambda}\left(\nabla_{\lambda} \ln \Omega\right)$ (here $\Omega=M$ ).

The above conclusion is true for any general spacetime. For the special case of stationary spacetimes however, we can have an interesting situation, namely that, even for an ordinary Killing vector of $g_{\mu \nu}$, we can find a conserved quantity. To see this clearly, we start from Eq. (20) and after a bit of algebra we arrive at

$$
\begin{aligned}
p^{\mu} \nabla_{\mu}\left(K_{v} p^{\nu}\right)= & K_{v} M u^{\mu} u^{\nu} \nabla_{\mu} M-K_{v} M^{2} h^{\mu \nu} \nabla_{\mu} \ln M \\
& +M^{2} u^{\mu} u^{\nu} \nabla_{\mu} K_{\nu} .
\end{aligned}
$$

Now suppose that in the particle's wave function, $\mathcal{R}\left(x^{\mu}\right)$ is independent of time so that the quantum potential term and hence $M$ are also time independent. In this case the vectors $\nabla_{\mu} M$ and $\nabla_{\mu} \log M$ have vanishing components along the $\mathrm{d} t$ direction. If the background spacetime is stationary, and the velocity is timelike, i.e., $u^{\mu}=(1,0,0,0)$, then for a vector field of the form $K^{\mu}=(1,0,0,0)$, the first two terms of Eq. (21) are identically zero (note that $h^{\mu \nu}$ is spacelike in this case). This means that for a stationary spacetime and time independent $\mathcal{R}$, once again $K_{\mu} p^{\mu}$ is conserved along the trajectory when the Killing equation is satisfied. This fact can be used to generate a quantum corrected version of any stationary spacetime (In [8] such correction to Schwarzschild solution was obtained by using the quantum Raychaudhuri equation).

As an immediate application of this, we can use the conformal transformation above find out a quantum corrected version of the Schwarzschild metric. Let us assume a simple stationary state wave function so that that its modulus is given by $\mathcal{R}(r)=N r \exp \left(-\frac{r^{2}}{2}\right)$, where $N$ is a normalization constant. Then we calculate the quantum potential associated with the wave function on Schwarzschild background

$f(\mathcal{R}(r))=\frac{\square \mathcal{R}}{\mathcal{R}}=\frac{1}{r^{3}}\left[r\left(r^{4}-5 r^{2}+2\right)-2 \mathcal{M}\left(r^{4}-4 r^{2}+1\right)\right]$,
$\mathcal{M}$ being the Schwarzschild mass. The conformal version of Schwarzschild solution is given by the metric

$$
\begin{aligned}
d \tilde{s}^{2}= & \left(m^{2}+\frac{1}{r^{3}}\left[2 \mathcal{M}\left(r^{4}-4 r^{2}+1\right)-r\left(r^{4}-5 r^{2}+2\right)\right]\right) \\
& \times\left\{-\left(1-\frac{2 \mathcal{M}}{r}\right) \mathrm{d} t^{2}+\left(1-\frac{2 \mathcal{M}}{r}\right)^{-1} \mathrm{~d} r^{2}+r^{2} d \Omega^{2}\right\} .
\end{aligned}
$$

This is the solution of the transformed field equation with $\tilde{T}_{\mu \nu}^{m}=0$. A particle will follow the geodesics of this metric. The matter part of EM tensor is zero due to the relation $\tilde{T}_{\mu \nu}^{m}=$ $M^{-2} T_{\mu \nu}^{m}$ discussed above.

\subsection{Absence of singularities in the conformal frame}

It was argued in [6] since the Bohmian trajectories cannot intersect each other, they do not form conjugate points and hence can avoid the usual singularities of GR. In this context, we ask the following question. In the conformally transformed frame there is no quantum force on the particle trajectory, then what happen to the conjugate points? Does the trajectory reach the singularities of the conformal frame $\tilde{g}_{\mu \nu}$ ?

To answer this question, we consider the following conjecture proposed long back in [22]. If a metric $g_{\mu \nu}$ is singular, we can always transform to the conformal frame $\tilde{g}_{\mu \nu}=\Omega^{2} g_{\mu \nu}$ which is non-singular and the singularities of the original metric show up as zeros of the conformal factor (as usual, the zeros of the conformal factor represent the boundaries of the spacetime). Keeping this conjecture in mind, the answer to the above question reduces to finding whether the conformal transformation of Eq. (16) is the required one to ensure the nonsingular nature of $\tilde{g}_{\mu \nu}$. If we consider a particle of mass $m$ moving on a geodesics of the metric $\tilde{g}_{\mu \nu}$, then in the frame $g_{\mu \nu}$ the particle's mass is time and position dependent (the modified mass in general depends on the nature of the conformal factor $\Omega$ ) and it moves on a nongeodesic. In [22], the author had shown by working out several examples, this variable particle mass of the transformed frame $g_{\mu \nu}$ should be the conformal factor to ensure the nonsingular nature of the metric $\tilde{g}_{\mu \nu}$. From Eq. (16) we see this is exactly the case when the particle moves in the quantum corrected spacetime $\tilde{g}_{\mu \nu}$.

This should be clear from the example we have worked out above. The original Schwarzschild metric is singular at $r \rightarrow 0$. But the Ricci curvature scalar $\tilde{R}$ of the metric $\tilde{g}_{\mu \nu}$ is non-singular in this limit, as can be checked explicitly, i.e., $\tilde{R}_{r \rightarrow 0}=$ constant. Also the conformal factor in the transformation equation in Eq. (23) is undefined in this limit. Thus if we start from the nonsingular frame $\tilde{g}_{\mu \nu}$ and work in a conformally related frame $g_{\mu \nu}$, the conformal factor vanishes 
precisely at $r=0$ making the transformation invalid at the singularity $r=0$.

Thus we conclude that the singularity is avoided by the particle trajectory in both frames related by a conformal transformation. However the mechanisms behind the avoidance are very different in the two frames. In the original classical background spacetime which contain genuine curvature singularities, a quantum particle trajectory never reaches theses singularities due to the force originating from the quantum potential [6]. On the other hand, the conformally related (and hence the quantum corrected) frame, where the particle motion is entirely classical, is free from the curvature singularities by construction, according to the conjecture proposed long back in [22]. Note also that the quantum potential and hence the conformal factor depends on the wave function, and thus it is not guaranteed that the transformed metric is generically singularity free. This general case will be investigated elsewhere.

\subsection{Problems with a conformally related frame}

So far we have shown by the transformation of Eq. (16), that it is possible to make the magnitude of $\tilde{p}_{\mu}$ a constant. By using the same transformation, can we write the continuity equation (second equation in Eq. (5)) in the desired form $\tilde{\nabla}_{\mu}\left(\rho \tilde{u}^{\mu}\right)=$ 0 ? The answer is no. To explain this, we shall first derive an important relation between the expansion scalars $(\theta$ and $\tilde{\theta}$ ) of two frames and the probability density $\rho$ (see Eq. (27) below).

A conformal transformation is equivalent to a corresponding change in the proper time $d \tau \rightarrow d \tilde{\tau}=\Omega d \tau$. In GR, under such conformal transformations, the change in the Christoffel symbols are given by $[17,21]$

$\delta \Gamma_{\mu \nu}^{\sigma}=2 \delta_{(\mu}^{\sigma} \partial_{\nu)} \ln \Omega-g_{\mu \nu} \partial^{\sigma} \ln \Omega$

Using this, one can check that the covariant derivative of the four velocity in the transformed frame is

$\tilde{\nabla}_{\mu} \tilde{u}_{v}=\Omega\left(\nabla_{\mu} u_{v}+g_{\mu \nu} u^{\alpha} \nabla_{\alpha} \ln \Omega-u_{\mu} \nabla_{\nu} \ln \Omega\right)$.

Taking the trace of this equation, we see that the expansion scalar $\theta$ of a geodesic congruence transforms under a conformal transformation as

$\tilde{\theta}=\Omega^{-1}\left(\theta+3 u^{\alpha} \nabla_{\alpha} \ln \Omega\right)$.

Using this relation we derive the following relation involving the probability density $\rho$,

$$
\begin{aligned}
& \tilde{\nabla}_{\mu}\left(\rho \tilde{u}^{\mu}\right)=\rho \tilde{\theta}+\tilde{u}^{\mu} \tilde{\nabla}_{\mu} \rho \\
& =\rho \Omega^{-1}\left(\theta+3 u^{\alpha} \nabla_{\alpha} \ln \Omega\right)+\Omega^{-1} u^{\mu} \nabla_{\mu} \rho .
\end{aligned}
$$

The criterion that in the transformed frame, the particle motion is a geodesic has already fixed the conformal factor $\Omega$ equal to $M$ up to a multiplicative constant. Now expanding the original conservation equation $\nabla_{\mu}\left(\rho M u^{\mu}\right)=0$ we have the relation

$\rho \theta=-\rho u^{\alpha} \nabla_{\alpha} \ln M-u^{\alpha} \nabla_{\alpha} \rho$.

Eliminating $\theta$ between Eqs. (27) and (28), we see that $\tilde{\nabla}_{\mu}\left(\rho \tilde{u}^{\mu}\right) \neq 0$. Thus transformation to a conformal frame gives rise to a wrong continuity equation. The reason for this is of course the fact that the momentum constrain relation has already fixed the conformal factor equal to $M$. As we shall show in sequel, for disformally related metrics, there is still enough freedom to fix both the problems.

A somewhat subtle point here is worth mentioning. Remember that we redefined the wavefunction as $\Phi=M \tilde{\Phi}$, so that the resulting action in Eq. (19) is more convenient to work with. As we have mentioned, in this redefinition, the norm of the wave function changes to $\tilde{\mathcal{R}}=M^{-1} \mathcal{R}$, and hence if one defines a transformed probability density $\tilde{\rho}=\tilde{\mathcal{R}}^{2}$ and demands that $\tilde{\nabla}_{\mu}\left(\tilde{\rho} \tilde{u}^{\mu}\right)$ is the quantity one should be looking for, we can see, by an analogous procedure as above that this quantity is indeed conserved, i.e., $\tilde{\nabla}_{\mu}\left(\tilde{\rho} \tilde{u}^{\mu}\right)=0$. But let us stress that this is not the continuity equation we are after, simply because the field redefinition (Weyl scaling) has nothing to do with the original conformal transformation $g_{\mu \nu} \rightarrow \tilde{g}_{\mu \nu}$, which is an actual change of the geometry itself, and not a change of coordinates or fields living in the spacetime [21]. The only purpose it serves (in this context) is to rewrite the action in a convenient form. Notice also that when $M^{2}<0$ the transformed density $(\tilde{\rho})$ is not even well defined in the transformed frame.

Apart from the wrong continuity equation, there is a further issue that is problematic in a conformally related frame. So far in our discussion of non geodesic motion, we have always consider a timelike trajectory for which $u^{\mu} u_{\mu}=-1$, but we can generalize the result for spacelike and null trajectories also. For the general case the Eq. (10) is given by

$u^{\mu} \nabla_{\mu} u^{v}=\frac{1}{2}\left(\alpha g^{\mu v}-u^{\mu} u^{v}\right) \nabla_{\mu} \ln \left(m^{2}-f(\mathcal{R})\right)$,

where $\alpha=-1,+1,0$, for time-like, space-like and null geodesics, respectively. Now for a massless particle, we put $m=0$, and since the term proportional to $u^{\mu} u^{\nu}$ can be absorbed in a re-parameterization of proper time, the only remaining term is proportional to $\alpha$, and hence the acceleration is zero for massless particle in both frames. So a natural question is, if null trajectories always move in geodesics, what happens to the singularity resolution argument for massless particles? That is, if the force due to the quantum poten- 
tial does not affect null trajectories, we ask why they do not form a caustic as in GR, and hence fall into a singularity?

We will next show that all the problems mentioned above are resolved by using disformal transformations instead. However before going into this, we will make an important simplification by assuming minimal coupling.

\subsection{Description in a minimally coupled background}

In analysing the motion of a quantum particle on a curved background so far we have worked by assuming that there exists a non-minimal coupling between the curvature scalar $R$ and the wavefunction describing the particle. Though sometimes presence of this term helps to make the analysis in the conformal frame simpler, it makes a nontrivial contribution to the Einstein equation, such that the metric in general will not be non-dynamical - as can be seen from Eq. (3). Usually this contribution is neglected (as was done in [6]) and $\Phi$ is assumed to be defined on a non-dynamical static background.

From now on we will instead start working in a frame where $\Phi$ is minimally coupled to the background [5]. Then the total action is similar to the one in Eq. (1) with $F(\Phi)=1$, and $\Phi$ satisfies the Klein-Gordon equation $\left(\square-m^{2}\right) \Phi=0$. The dispersion relation now changes to

$p_{\mu} p^{\mu}=-m^{2}+\frac{\square \mathcal{R}}{\mathcal{R}}=M^{2} u_{\mu} u^{\mu}$,

which indicates that a quantum particle will follow a non geodesics motion in this frame as given in Eq. (10), with $\epsilon=0$. We can always transform to a conformal frame, where the motion of the particle will be on a geodesic and quantum effects are included in the energy momentum tensor, but in that frame there is a non-minimal coupling between the curvature scalar and the redefined wavefunction. ${ }^{3}$

The advantage of this approach compared to the previous one is that, this picture is more in line with the scalar tensor theories of gravity $[17,18]$. There, in the Jordan frame, a test particle follows the geodesic equation. On the other hand, in the conformally related Einstein frame, the scalar field couples to matter, and the test particle follows non-geodesic motion, as the interaction between the scalar field and matter causes the particle to feel an additional force [18]. Also in this approach, since in the original frame, the quantum mass given by $M=\sqrt{m^{2}-f(\mathcal{R})}$ is independent of the coupling constant $\epsilon$, its nature is unambiguous, i.e., if $f(\mathcal{R})>m^{2}$, then the quantum mass is imaginary, otherwise it is real. If we had started with a nonminimal coupling, this depends on the constant $\epsilon$ as in the previous sections. As we will

\footnotetext{
${ }^{3}$ For a massless particle one can make the action and hence the KleinGordon equation conformally invariant by choosing $\epsilon=1 / 6$ in 4 spacetime dimensions. But for massive particles, with which we shall mostly work with, this invariance is lost.
}

momentarily see, this property helps to solve the problem of indefiniteness of quantum mass uniquely in this frame, using a disformal transformation.

All the calculations in the rest of the paper are performed assuming a minimal coupling. When there is non-minimal coupling between gravitational and particle degrees of freedom, our calculations can be generalized with some minor modifications, but in the transformed frame it is rather difficult to see the quantum effects in the metric through calculating the EM tensor, because there will be terms coming from the non-minimal coupling also (with minimal coupling, the last two terms in the right hand side of Eq. (3) are zero). In short, when there is already a coupling between classical background and the quantum particle, the process of incorporating quantum effects in background geometry loses some of its meaning because from the start the classical aspects of the spacetime and the quantum aspects of the particle are related. For the non-minimal case they separate to begin with.

\section{Bohmian trajectory on a physical geometry}

So far we have assumed a description of gravity where gravitational and particle dynamics take place in conformally related global Riemannian spacetimes, called gravitational and physical geometries respectively. But as we have pointed out in the introduction, the relation between these two geometries can be more general than the conformal transformation. It was shown in [1], by assuming the physical geometry (on which the matter dynamics takes place) to be Finslerian (instead of Riemannian), and using arguments based on the weak equivalence principle and causality, that in the most general case, both the gravitational and the physical geometries have to be Riemannian and that in general such geometries are related to each other by a disformal transformation. It is thus natural to ask what are the consequences of quantum motion in terms of Bohmian mechanics in this more general case. This is the topic we discuss in this section.

\subsection{Disformal transformations}

Two metrics $g^{*}$ and $g$ (and their inverses) are said to be related to each other by the a disformal transformation, if the relation between them is given by $[1,16]$

$$
\begin{aligned}
g_{\mu \nu}^{*} & =\Omega^{2}(\phi, X) g_{\mu \nu}-\alpha \mathcal{B}(\phi, X) \phi_{\mu} \phi_{\nu}, \\
g^{* \mu \nu} & =\Omega^{-2}\left[g^{\mu \nu}+\alpha \frac{\mathcal{B}}{\Omega^{2}-2 X \mathcal{B}} \phi^{\mu} \phi^{\nu}\right], \\
X & =-\frac{1}{2} g^{\mu \nu} \phi_{\mu} \phi_{\nu},
\end{aligned}
$$

where $\alpha=0, \pm 1$, both $\Omega$ and $\mathcal{B}$ are arbitrary real functions of a scalar field $\phi$, and we have used the notation $\phi_{\mu}=\nabla_{\mu} \phi$ to 
denote the normal vector to a $\phi=$ const hypersurface. Note that all indices of $\phi_{\mu}$ are raised and lowered by the metric $g_{\mu \nu}$. We will denote all the tensor quantities with respect to $g_{\mu \nu}^{*}$ with a superscript “ $*$ ".

If we consider the motion of a particle moving on a timelike trajectory we can choose the normal vectors $\phi_{\mu}$ to be hypersurface orthogonal velocity vector $v^{\mu}$ of the trajectories, and in this case $\alpha=-1$ (though in the calculations below we will keep $\alpha$ to generalize the results to a spacelike trajectories also). The identification of $\phi_{\mu}$ with velocity is a choice, and as long as we are considering the motion along timelike (or spacelike) trajectory this is a good choice, but for null trajectories we will make a different choice because in general via a disformal transformation, unlike a conformal one, a null geodesic can map to a non-geodesic trajectory (this will be crucial in our arguments in Sect. 4.2). Thus the form of the disformal transformation and its inverse that we will consider for now are the following [25]

$$
\begin{aligned}
g_{\mu \nu}^{*} & =\Omega^{2}(x) g_{\mu \nu}-\alpha \mathcal{B}(x) v_{\mu} v_{\nu}, \\
g^{* \mu \nu} & =\Omega^{-2}\left[g^{\mu \nu}+\alpha\left(\frac{\mathcal{B}}{\Omega^{2}-\mathcal{B}}\right) v^{\mu} v^{\nu}\right] .
\end{aligned}
$$

This transformation is equivalent to a transformation of the proper time [16]

$$
\begin{aligned}
d \tau^{* 2} & =-g_{\mu \nu}^{*} d x^{\mu} d x^{\nu}=-\left[\Omega^{2} g_{\mu \nu}-\alpha \mathcal{B} v_{\mu} v_{\nu}\right] d x^{\mu} d x^{\nu} \\
& =\left[\Omega^{2}+\alpha \mathcal{B}\right] d \tau^{2}
\end{aligned}
$$

Now, the tangent vector to a particle trajectory is $v^{\mu}=$ $d x^{\mu} / d \tau$. With respect to $g_{\mu \nu}^{*}$, this vector is defined as $v^{* \mu}=d x^{\mu} / d \tau^{*}$. From the above relations we can find out the followings

$v_{\mu}^{*}=\sqrt{\mathcal{F}} v_{\mu}, \quad v^{* \mu}=g^{* \mu v} v_{v}^{*}=\mathcal{F}^{-1 / 2} v^{\mu}$,

$\mathcal{F}=\Omega^{2}+\alpha \mathcal{B}, g_{\mu \nu}^{*} v^{* \mu} v^{* \nu}=\alpha$.

As can be seen, the vector $v^{* \mu}$ is normalized with respect to the transformed metric $g^{* \mu \nu}$.

\subsection{Relation between acceleration vectors and particle motion}

Using the formulas given above, we can now establish the required relation between acceleration vectors $a^{v}=v^{\mu} \nabla_{\mu} v^{v}$ and $a^{* \nu}=v^{* \mu} \nabla_{\mu}^{*} v^{* \nu}$ of two metrics, where $\nabla_{\mu}^{*}$ is the covariant derivative with respect to $g_{\mu \nu}^{*}$. First, we write down the known relation between the quantities $\Gamma_{\mu \nu}^{* \lambda} v_{\lambda}^{*}$ and $\Gamma_{\mu \nu}^{\lambda} v_{\lambda}$ (see [25] for details)

$$
\begin{aligned}
\Gamma_{\mu \nu}^{* \lambda} v_{\lambda}^{*}= & \sqrt{\mathcal{F}} \Gamma_{\mu \nu}^{\lambda} v_{\lambda}+\frac{1}{2 \sqrt{\mathcal{F}}}\left[\alpha v_{\mu} v_{\nu} v^{\alpha} \nabla_{\alpha} \mathcal{F}-h_{\mu \nu} v^{\alpha} \nabla_{\alpha} \Omega^{2}\right. \\
& \left.-2 \mathcal{B} K_{\mu \nu}+2 v_{(\mu} h_{\nu)}^{\alpha} \nabla_{\alpha} \mathcal{F}\right]
\end{aligned}
$$

where $\Gamma_{\mu \nu}^{\lambda}$ are the connection coefficients, and we have defined the extrinsic curvature in standard fashion, as

$K_{\mu \nu}=h_{\mu}^{\alpha} \nabla_{\alpha} u_{\nu}=\nabla_{\mu} v_{\nu}-\alpha a_{\nu} v_{\mu}$.

Substituting Eq. (35) in the formula $\nabla_{\mu}^{*} v_{v}^{*}=\partial_{\mu} v_{\nu}^{*}-\Gamma_{\mu \nu}^{* \lambda} v_{\lambda}^{*}$, after a few steps of straightforward algebra, we get the following relation between covariant derivative of a vector with respect to both metrics

$$
\begin{aligned}
\nabla_{\mu}^{*} v_{\nu}^{*}= & \sqrt{\mathcal{F}}\left(\nabla_{\mu} v_{v}+\frac{v^{\alpha} \nabla_{\alpha} \Omega^{2}}{2 \mathcal{F}} h_{\mu v}+\frac{\mathcal{B}}{\mathcal{F}} K_{(\mu v)}+\frac{v_{\nu} \nabla_{\mu} \mathcal{F}}{2 \mathcal{F}}\right. \\
& \left.-\frac{\alpha v_{\mu} v_{\nu} v^{\alpha} \nabla_{\alpha} \mathcal{F}}{2 \mathcal{F}}-\frac{1}{\mathcal{F}} v_{(\mu} h_{\nu)}^{\alpha} \nabla_{\alpha} \mathcal{F}\right)
\end{aligned}
$$

Note that the last three terms in Eq. (37) are missing in [25]. But these terms are essential to establish a relation between the acceleration vectors, in which case both the second and third terms vanish. Multiplying both sides of Eq. (37) by $v^{* \mu}$, and after a bit of manipulation we get the following simplified version of the desired relation between the acceleration vectors

$a_{\mu}^{*}=a_{\mu}-\frac{1}{2 \mathcal{F}} \alpha h_{\mu}^{\nu} \nabla_{\nu} \mathcal{F}=a_{\mu}-\frac{1}{2} \alpha h_{\mu}^{\nu} \nabla_{\nu} \ln \mathcal{F}$.

It is worth remarking that due to absence of the terms in Eq. (37) mentioned above in the corresponding formula in [25], the author has concluded that the accelerations are equal. But accelerations of conformally (and also disformally) related frames are not equal, at least in GR. This is the root of the problem that one faces when constructing conformally invariant observables in gravity theories with symmetric connection (see the discussion is Sect. 5 below).

Note also that we shall recover all the results of previous sections on the conformal transformation by putting $\mathcal{B}=0$ in the above expressions. As is evident from this equation, two vectors $a_{\mu}^{*}$ and $a_{\mu}$ cannot be equal to each other unless the difference of $\Omega$ and $\mathcal{B}$ is a constant. This in turn means that two velocity vectors $v_{\mu}$ and $v_{\mu}^{*}$ cannot represent geodesics simultaneously. Indeed from Eq. (38), we glean that

$a_{\mu}^{*}=v^{* \nu} \nabla_{\nu}^{*} v_{\mu}^{*}=0 \Longrightarrow a_{\mu}=v^{\nu} \nabla_{\nu} v_{\mu}=\alpha h_{\mu}^{\nu} \nabla_{\nu} \ln \sqrt{\mathcal{F}}$.

Thus in the physical geometry, if we consider the geodesic motion of a particle of mass $m$, then in the disformally related gravitational geometry, this represents an accelerated motion with the force being perpendicular to the four-velocity.

Now if we consider a quantum particle of mass $m>0$ in the gravitational geometry moving along a Bohmian trajectory so that it is acted upon by force originated due to the quantum potential then the norm of it's 4-momentum satisfy relation Eq. (30). If we represent it's acceleration by 
the 2nd equation of Eq. (39) with $\alpha=-1$, in disformally related geometry this corresponds to a force free motion with the quantum effects taken in the modified metric $g_{\mu \nu}^{*}$ of the physical geometry. We can easily determine the desired relation between the transformation factors $\Omega$ and $\mathcal{B}$ in terms of the modulus of the wave function $\mathcal{R}$, by comparing Eqs. (39) and (10) to be ${ }^{4}$,

$\mathcal{F}=\Omega^{2}-\mathcal{B}=\left(1-f(\mathcal{R}) / m^{2}\right)$.

We have put $\epsilon=0$ because we have assumed no coupling between the curvature and the wavefunction $\Phi$. Note that the constraint relation of Eq. (30) is not enough to uniquely determine the both the transformation factors in Eq. (40), and below we will see that a second relation between the transformation factors arise through the continuity equation.

It is also convenient to write down the corresponding particle actions in this case. Given the constraint relation of Eq. (30), by following the procedure outlined previously, we can derive the action of a particle moving along the Bohmian trajectory in the frame $g_{\mu \nu}$. This is just given by Eq. (17) with $\epsilon=0$. Now using the rules of active transformation i.e., substituting $g_{\mu \nu}$ in terms of $g_{\mu \nu}^{*}$ and $v^{* \mu}$ from Eq. (32) we see that the action

$$
S_{m}\left[g_{\mu \nu}, \gamma\right]=-\int_{\gamma} \sqrt{m^{2}-f(\mathcal{R})} \sqrt{-g_{\mu \nu} v^{\mu} v^{v}} d \tau
$$

gets transformed to the action of a particle of unit mass

$$
\begin{aligned}
S_{m}^{D}\left[g_{\mu \nu}^{*}, \gamma\right] & =-\int_{\gamma} \sqrt{-\Omega^{-2}\left[g_{\mu \nu}^{*}+\frac{\alpha \mathcal{B}}{\mathcal{F}} v_{\mu}^{*} v_{\nu}^{*}\right] \mathcal{F} v^{* \mu} v^{* \nu}} d \tau^{*} \\
& =-\int_{\gamma} \sqrt{-g_{\mu \nu}^{*} v^{* \mu} v^{* v}} d \tau^{*} .
\end{aligned}
$$

Here in the first step, we have used the previous identification of Eq. (40), and also substituted $d \tau$ in terms of $d \tau^{*}$, and the trick in the second step is to write one set of $g_{\mu \nu}^{*} v^{* \mu} v^{* \nu}$ as $\alpha$ so that it cancels with the other term. With these particle actions, it is easy to check that they satisfy the respective equations in Eq. (39), thus confirming our conclusions. Note that here (and in the previous section during the discussions on the conformal version) we have taken an active point of view in transforming the action, namely we have taken the functional form of the action same as before (i.e., the action of Eq. (8) with the Lagrangian in Eq. (9)), and replaced the original metric by the transformed one. This procedure naturally leads to a value of the action different from the previous one. On the other hand, if we want to keep the form of the action unchanged then the passive transformation should be used. In this paper we shall always use the active point of view

\footnotetext{
${ }^{4}$ As before we will neglect the unimportant factor of $m^{2}$ and will take $\mathcal{F}=M^{2}$ below.
}

unless otherwise specified (see $[20,26]$ for transformation with passive point of view).

We can also write down the modified Raychaudhuri equation and the deviation equation in this case also following the lines described in Sect. 2.1 for the conformal frame. One can check these are still given by Eqs. (12) and (14) respectively, with $L=-\frac{1}{2} \ln \mathcal{F}$.

\section{Advantages of disformal transformations}

Now that we have the characterization of a quantum particle in both the gravitational and the physical geometry, and have shown that like the conformal transformation, a disformal transformation can be successfully used to incorporate the quantum effects in the geometry, one can ask where exactly it differs from the usual picture of conformal transformation and what are the advantages of this identification over the conformal transformation, if any. In this section we shall show that a disformal transformation has its advantages, namely it can solve the problems addressed in Sect. 2.5.

\subsection{Continuity equation fixes the disformal transformation completely}

As we discussed in Sect. 2.5, the conformal transformation with the quantum mass squared as the conformal factor can not give us the right continuity equation. Here we shall show by doing a disformal transformation of the form Eq. (32), it is possible solve this problem thereby completely fixing both the transformation factors $\Omega^{2}$ and $\mathcal{B}$.

We start by writing down the disformal version of the Eq. (26) that relates the expansion scalar in both frames (see [25] for the derivation)

$\theta^{*}=\mathcal{F}^{-1 / 2}\left(\theta+3 v^{\alpha} \nabla_{\alpha} \ln \Omega\right)$

Then, as before, we have the analogue of Eq. (27) given by

$$
\begin{aligned}
\nabla_{\mu}^{*}\left(\rho v^{* \mu}\right)= & \rho \theta^{*}+v^{* \mu} \nabla_{\mu}^{*} \rho=\rho \mathcal{F}^{-1 / 2}\left(\theta+3 v^{\alpha} \nabla_{\alpha} \ln \Omega\right) \\
& +\mathcal{F}^{-1 / 2} v^{\mu} \nabla_{\mu} \rho .
\end{aligned}
$$

Now using Eq. (28) to eliminate $\theta$ from this equation we get

$\nabla_{\mu}^{*}\left(\rho v^{* \mu}\right)=\rho \mathcal{F}^{-1 / 2}\left(-v^{\alpha} \nabla_{\alpha} \ln M+v^{\alpha} \nabla_{\alpha} \ln \Omega^{3}\right)$.

The requirement that in the disformally transformed frame $\nabla_{\mu}^{*}\left(\rho v^{* \mu}\right)=0$ is the correct continuity equation indicates the left hand side to be zero and thus fixes the conformal 
factor $^{5}$ to be $\Omega^{3}=M$. This, together with the requirement of Eq. (40) also fixes the disformal factor $\mathcal{B}=M^{2 / 3}-M^{2}$.

\subsection{Motion of a photon along physical null trajectory}

As was pointed out towards the end of Sect. 2.5, massless particles ${ }^{6}$ cause a problem in the singularity resolution argument because they do not experience any force in frames related by conformal transformations. For the case of frames related by disformal transformations (Eq. (32) considered in the previous section), the same problem arises because we see from the corresponding relation Eq. (39), the same conclusion remains valid, i.e., the extra force term is zero for null trajectories.

We shall argue below that we can cure this problem in the context of disformal transformation, by remembering that, one can make a disformal transformation more general than the one written in Eq. (32), where the extra piece need not be necessarily pointing along the direction of the tangent of a particle trajectory. This transformation precisely is what given in Eq. (31), where the non conformal piece is along a direction specified by the $\phi=$ const hypersurface, which we can choose to be different from the four velocity. This is the advantage of disformal transformations over conformal transformations, namely that one can make a massless particle move on a non geodesic motion by going to a frame where square of its four momentum is non zero. Below we shall consider a simple form of Maxwell's equation to show clearly how this can be done (see [16] where the transformation of the Maxwell equations under a disformal transformation are considered by assuming the geometrical optics approximation. However we do not make any such approximation).

Let us consider the motion of a photon in the gravitational geometry (represented by a vector field $A_{\mu}$ ) described by the following Maxwell equations

$\nabla^{\mu} \nabla_{\mu} A_{\nu}-\nabla^{\mu} \nabla_{\nu} A_{\mu}=0$

where we have put all the source terms to zero. Now, using the Ricci identity which relates the commutator of the covariant derivative of a vector field to the Riemann tensor,

$\nabla_{\mu} \nabla_{\nu} A^{\alpha}-\nabla_{\nu} \nabla_{\mu} A^{\alpha}=R_{\beta \mu \nu}^{\alpha} A^{\beta}$,

in the contracted form, and imposing the Lorentz gauge condition $\nabla^{\mu} A_{\mu}=0$, we write the above equation as

$\nabla^{\mu} \nabla_{\mu} A_{\nu}-R_{v}^{\mu} A_{\mu}=0$.

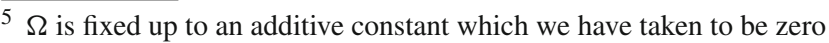

${ }^{6}$ Note that by a massless particle, we mean a particle with zero classical mass $(m)$, not zero quantum mass $(M)$. More precisely what we mean by massless particle here are the particles which moves along the trajectory $u_{\mu} u^{\mu}=0$ and hence $p_{\mu} p^{\mu}=0$.
As before we write $A_{\mu}$ in the polar form

$A_{\mu}=\mathcal{C}_{\mu}(x) e^{i \mathcal{S}(x)}$,

and substitute in the Maxwell's equation. After separating the real and imaginary parts, and identifying $\mathcal{K}_{\mu}=\nabla_{\mu} \mathcal{S}$ as the wave vector of the photon, we get the following equations

${ }^{\mu \nu} \mathcal{K}_{\mu} \mathcal{K}_{\nu}=\frac{\mathcal{C}^{v}}{\mathcal{C}^{2}} \nabla^{\mu} \nabla_{\mu} \mathcal{C}_{\nu}-R_{\nu}^{\mu} \mathcal{C}_{\mu} \mathcal{C}^{v} \equiv \mathcal{H}(\mathcal{C})$,

$\nabla_{\mu}\left(\mathcal{C}^{2} \nabla^{\mu} \mathcal{S}\right)=0$

where $\mathcal{C}^{2}=g^{\mu \nu} \mathcal{C}_{\mu} \mathcal{C}_{v}$ denotes the magnitude of the vector $\mathcal{C}_{\mu}$. The first relation gives the magnitude of the wave vector, with $\mathcal{H}(\mathcal{C})$ denoting the quality analogous to quantum potential in this case, and the second one is essentially the conservation equation. As can be anticipated from Eq. (50), since in this frame, motion of the photon is represented by the non null vector $\mathcal{K}_{\mu}$, the motion of photon is along a non geodesic trajectory.

Let us now apply the disformal transformation (Eq. (31) with $\alpha=-1$ ) and see if we can make the photon motion a geodesic in the transformed frame. In doing so, the first thing to notice is that, since the transformation functions in Eq. (31) are real, the phase factor $\mathcal{S}$ and hence the wave vector $\mathcal{K}_{\mu}=\nabla_{\mu} \mathcal{S}$ is equal in both the frames. Then, using the inverse relation of Eq. (31), the left hand side of first equation of Eq. (50) reduces to

$g^{\mu \nu} \mathcal{K}_{\mu} \mathcal{K}_{v} \rightarrow \Omega^{2} g^{* \mu v} \mathcal{K}_{\mu} \mathcal{K}_{v}+\frac{\mathcal{B}}{\Omega^{2}-2 X \mathcal{B}}\left(\phi^{\mu} \mathcal{K}_{\mu}\right)^{2}$

Now it is easy to see by comparing Eqs. (50) and (51), that if we want to make $\mathcal{K}_{v}$ null in the transformed frame, then we have to choose the transformation functions such that

$$
\frac{\mathcal{B}}{\Omega^{2}-2 X \mathcal{B}}\left(\phi^{\mu} \mathcal{K}_{\mu}\right)^{2}=\frac{\mathcal{C}^{\nu}}{\mathcal{C}^{2}} \nabla^{\mu} \nabla_{\mu} \mathcal{C}_{\nu}-R_{\nu}^{\mu} \mathcal{C}_{\mu} \mathcal{C}^{\nu}=\mathcal{H}(\mathcal{C})
$$

Of course, this single equation does not completely determine the disformal transformation specified by $\Omega, \mathcal{B}, \phi^{\mu}$. We can have another relation from the continuity equation. However unlike the previous case for massive particles, these two equations (Eq. (52) and the one obtained from the continuity equation) are not enough for fixing both the transformation factors $\Omega$ and $\mathcal{B}$ and the direction of the disformal vector specified by components of $\phi_{\mu}$ uniquely. We also have to make some choice of the factor $\Omega$ (such as pure the disformal transformation with $\Omega=$ constant) and/or of the disformal vector (such as timelike $\left(\phi_{\mu} \phi^{\mu}=-1\right.$ ) or null disformal transformation $\left(\phi_{\mu} \phi^{\mu}=0\right)$ ). The description of the photon's motion with such explicit choices are left for a future work. 
The equation of motion derived from Eq. (50) by taking covariant derivative of both side is the non geodesic equation

$$
\mathcal{K}^{\mu} \nabla_{\mu} \mathcal{K}_{\nu}=\frac{1}{2} \nabla_{\nu}\left[\frac{\mathcal{B}}{\Omega^{2}-2 X \mathcal{B}}\left(\phi^{\mu} \mathcal{K}_{\mu}\right)^{2}\right]=\frac{1}{2} \nabla_{\nu} \mathcal{H}(\mathcal{C}),
$$

which, as can be checked, under a disformal transformation satisfying Eq. (52) transforms to the geodesic equation

$\mathcal{K}^{* \mu} \nabla_{\mu}^{*} \mathcal{K}_{\nu}^{*}=0$

Thus the motion of a photon in a gravitational geometry follows a non null trajectory (the null trajectories $d s^{2}=$ $g_{\mu \nu} d x^{\mu} d x^{\nu}=0$ in the gravitational geometry are followed by the gravitons [1]) and hence its motion is nongeodesic, acted upon by the force resembling that of due to the quantum potential (see Eqs. (50) and (53) respectively). But this motion in a disformally related physical geometry follows a null trajectory, $d s^{* 2}=g_{\mu \nu}^{*} d x^{\mu} d x^{\nu}=0$ of that geometry, where $\mathcal{H}(\mathcal{C})$ determines the required transformation factors. As we have shown in Sect. 2.3, $p_{\mu} K^{\mu}$ is a conserved quantity along the non geodesic motion, provided that $K^{\mu}$ is a conformal Killing vector i.e., a Killing vector of a conformally related metric. By using an analogous procedure, it is easy to see that $\mathcal{K}_{\mu} K^{\mu}$ is a conserved quantity along the photon trajectory if it satisfies the equation $\nabla_{(\mu} K_{\nu)}=-2 g_{\mu \nu} K^{\lambda} \nabla_{\lambda}(\ln \mathcal{H}(\mathcal{C}))$, and this quantity should be used if one wants to study the deflection of light in gravitational field.

\subsection{Problem of definiteness of mass}

When one uses the standard Klein-Gordon equation to perform a Bohmian treatment, one gets the resulting quantum Hamilton-Jacobi equation of Eq. (30). The right side of this equation, interpreted as the mass square (denoted as $M^{2}$ ) is not always positive definite, and hence the theory can have tachyonic solutions (see for example [3]). As we have mentioned before, one motivation for transforming to a conformal frame to describe the quantum motion of a particle satisfying the Klein-Gordon equation is to avoid this problem, so that in the transformed frame the particle has a positive definite mass. But also as pointed out before, this comes with other problems such as the wrong continuity equation and the problem with massless particles. Most importantly when the quantum mass $M$ is imaginary, the conformal factor is itself negative and hence the transformation is not well defined.
In Sect. 4.1, we have shown that for a massive particle ${ }^{7}$ by transforming to a disformal frame where two metrics are related by Eq. (32), with the transformation factors given in terms of the quantum mass by the relations

$\Omega^{2}=M^{2 / 3}, \quad \mathcal{B}=M^{2 / 3}-M^{2}, \quad M(x)=\sqrt{\left(m^{2}-f(\mathcal{R})\right)}$,

one could achieve a consistent continuity equation for the motion of the quantum particle. However the same transformation does not solve the problem of definiteness of mass. If the quantum mass is imaginary, then the real root of the conformal factor $\Omega^{2}=M^{2 / 3}$ in Eq. (55) becomes negative, so that the disformal transformation used earlier becomes undefined. ${ }^{8}$

In this subsection, we shall show that a possible way out of this problem is once again to return to the general form of the disformal transformation in Eq. (31). However, before doing that let us try to locate the problem in the previous setting. When the mass squared is negative, we can express the quantum mass formally as $M=|M| e^{i \pi / 2}$. Now from the continuity equation in the transformed frame, we see that the extra phase of $\pi / 2$ being a constant, does not affect this equation

$\nabla_{\mu}^{*}\left(\rho v^{* \mu}\right)=\rho \mathcal{F}^{-1 / 2}\left(-v^{\alpha} \nabla_{\alpha} \ln |M|+v^{\alpha} \nabla_{\alpha} \ln \Omega^{3}\right)$.

Thus by taking $\Omega^{2}=|M|^{2 / 3}$, we can still satisfy the continuity equation in the transformed frame, even when $M$ is imaginary. Looking at the acceleration in Eq. (10) (with $\epsilon=0$ ), we could similarly argue that, by choosing $\sqrt{\mathcal{F}}=\sqrt{\Omega^{2}-\mathcal{B}}=|M|$, this equation can be satisfied as well, since the constant phase factor again does not contribute due to the derivative. However such a choice does not satisfy the correct dispersion relation $g^{* \mu v} p_{\mu}^{*} p_{\nu}^{*}=-1$, because under such a transformation, the required relation

\footnotetext{
${ }^{7}$ For the description of massless particle one has to use a more general transformation of Eq. (31). In this subsection, we shall concentrate on the case of massive particles only.

${ }^{8}$ In the non-minimally coupled frame, the quantum mass has an extra factor of $\epsilon R$ (see Eq. (7) above). Can this factor make quantum mass in this frame positive definite? Assuming $\epsilon>0$, the answer will depend on the sign of the Ricci scalar. When $R<0$, the $\epsilon R$ term can not make the quantum mass positive definite and for $R>0$ this can make $M$ come out to be positive depending on its relative magnitude with the quantum potential. As mentioned in Sect. 2.6, this is one of the advantages of working with minimal coupling - the nature of $M$ does not depends on the coupling $\epsilon$.
} 


$$
\begin{aligned}
g^{\mu \nu} p_{\mu} p_{v} & =|M|^{2} \rightarrow\left[\Omega^{2} g^{* \mu \nu}+\left(\frac{\mathcal{B}}{\Omega^{2}-\mathcal{B}}\right) v^{\mu} v^{v}\right] \\
p_{\mu} p_{v} & \equiv-\Omega^{2}+\left(\frac{-\mathcal{B}|M|^{2}}{\Omega^{2}-\mathcal{B}}\right) \neq|M|^{2},
\end{aligned}
$$

is not satisfied when we choose $\Omega^{2}-\mathcal{B}=|M|^{2}$. This is the real problem, namely to satisfy the correct dispersion relation when quantum mass is imaginary. Indeed the Bohmian treatment of Klein-Gordon equation gives the dispersion relation, and not the acceleration equation (see Eq. (6)). In the process of taking the covariant derivative, the imaginary factors are "lost" in both Eq. (56) and the continuity equation, and one can reach wrong conclusions by considering them. Thus our primary focus will be on the dispersion relation.

We begin the general case by writing above Eq. (57) for such a transformation in Eq. (31). The relation we want to satisfy is the following

$$
\begin{aligned}
g^{\mu \nu} p_{\mu} p_{v} & =|M|^{2} \rightarrow\left[\Omega^{2} g^{* \mu \nu}+\left(\frac{\mathcal{B}}{\Omega^{2}-2 \mathcal{B} X}\right) \phi^{\mu} \phi^{\nu}\right] \\
p_{\mu} p_{v} & \equiv-\Omega^{2}+\left(\frac{-\mathcal{B D} \mathcal{D}^{2}|M|^{2}}{\Omega^{2}-2 \mathcal{B} X}\right)=|M|^{2}
\end{aligned}
$$

where $\mathcal{D}=\phi^{\mu} v_{\mu}$ denotes the projection of the vector $\phi^{\mu}$ along the particle 4-velocity.

For our purpose in this subsection, it is sufficient to consider the so called pure disformal transformation, where both the conformal and disformal factors are set to a constant (denoted as $\Omega_{0}^{2}$ and $\mathcal{B}_{0}$ respectively, with $\Omega_{0}^{2}>0$ ). Thus the transformed metric and it's inverse are given respectively by

$$
\begin{aligned}
g_{\mu \nu}^{\star} & =\Omega_{0}^{2} g_{\mu \nu}+\mathcal{B}_{0} \phi_{\mu} \phi_{\nu}, \\
g^{\star \mu \nu} & =\Omega_{0}^{-2}\left[g^{\mu \nu}-\frac{\mathcal{B}_{0}}{\Omega_{0}^{2}-2 X \mathcal{B}_{0}} \phi^{\mu} \phi^{\nu}\right], \\
X & =-\frac{1}{2} g^{\mu \nu} \phi_{\mu} \phi_{\nu} .
\end{aligned}
$$

All the transformed quantities are denoted by an overhead star. Here the 4-vector $\phi^{\mu}$, having components $\left(\phi^{0}, \phi^{1}\right.$, $\left.\phi^{2}, \phi^{3}\right)$, determine the direction of the disformal transformation and is to be determined form the correct transformation of the dispersion relation and the continuity equation. The above transformation corresponds to a change of proper time $d \tau^{\star 2}=\beta^{2} d \tau^{2}$ with $\beta=\left(\Omega_{0}^{2}-\mathcal{B}_{0} \mathcal{D}^{2}\right)^{1 / 2}$. Then Eq. (58) gives the first constraint relation to be

$$
\begin{aligned}
& \Omega_{0}^{2}+\left(\frac{\mathcal{B}_{0} \mathcal{D}^{2}|M|^{2}}{\Omega_{0}^{2}-2 \mathcal{B}_{0} X}\right)+|M|^{2}=0, \text { with } \\
& \mathcal{D} \neq 0 \text { unless }|M|=\text { constant. }
\end{aligned}
$$

The continuity equation gives another constraint. To determine this, we start by writing down the transformation rule of the covariant derivative. In the most general case, the formula for the change of the Christoffel symbols are quite complicated (see for example $[16,27,28]$ ), but for our case of the transformation in Eq. (59), the transformation relation of the covariant derivative of a vector simplifies to the following

$\nabla_{\mu}^{\star} v^{\nu}=\nabla_{\mu} v^{\nu}+C_{\nu \alpha}^{\mu} v^{\alpha}$ where $C_{\nu \alpha}^{\mu}=\left(\frac{\mathcal{B}_{0}}{\Omega_{0}^{2}-2 X \mathcal{B}_{0}}\right) \phi^{\mu} \nabla_{\alpha} \phi_{\nu}$

Writing the left side in terms of transformed velocity $v^{\star v}$, and simplifying, we arrive at

$\nabla_{\mu}^{\star} v^{\star \nu}=\beta^{-1}\left(\nabla_{\mu} v^{\nu}+C_{\nu \alpha}^{\mu} v^{\alpha}-v^{\nu} \nabla_{\mu} \beta\right)$

This gives the desired relation between the expansion scalars (compare with Eqs. (26) and (43) which are the analogous relations for the conformal transformation and disformal transformation along the velocity vector respectively)

$\theta^{\star}=\beta^{-1}\left(\theta+C_{\nu \alpha}^{\nu} v^{\alpha}-v^{\nu} \nabla_{\nu} \beta\right)$.

The continuity equation can now be expanded to give

$$
\begin{aligned}
\nabla_{\mu}^{\star}\left(\rho v^{\star \mu}\right)= & \rho \theta^{\star}+v^{\star \mu} \nabla_{\mu}^{\star} \rho=\rho \beta^{-1}\left(\theta+C_{\nu \alpha}^{v} v^{\alpha}-v^{\nu} \nabla_{\nu} \beta\right) \\
& +\beta^{-1} v^{\mu} \nabla_{\mu} \rho .
\end{aligned}
$$

As before, eliminating $\theta$ from this equation and demanding that continuity equation $\nabla_{\mu}^{\star}\left(\rho v^{\star \mu}\right)=0$ is satisfied, we get the second constraint

$C_{\nu \alpha}^{v} v^{\alpha}-v^{\nu} \nabla_{v} \beta-v^{\alpha} \nabla_{\alpha} \ln |M|=0$.

Once again the pure phase factor does have any effect in the continuity equation, so that components of $\phi^{\mu}$ are real. If we choose the vector field $\phi^{\mu}$ such that, given the quantum mass both the constraint Eqs. (60) and (65) are satisfied, then in the transformed frame $\left(g_{\mu \nu}^{\star}\right)$ the particle has unit mass, thus solving the problem of imaginary quantum mass in the original frame. Obviously the two constraints of Eqs. (60) and (65) cannot determine all the four components of $\phi_{\mu}$, and we have to make some choices.

Below we shall briefly sketch such a procedure when the quantum mass of the particle $(M)$ moving in the flat spacetime is imaginary and is a function of the radial coordinate $r$ only. ${ }^{9}$ We consider the vector field $\phi^{\mu}$ to be of the form $\left(1, \phi^{0}(r), 0,0\right)$ and the particle 4 velocity is $v^{\mu}=(1,0,0,0)$

\footnotetext{
9 This mass can corresponds to a stationary state solution of the KleinGordon equation for which the quantum potential is time independent.
} 
so that $\mathcal{D}=1$. Then, as can be checked, the continuity equation is identically satisfied and Eq. (60) gives the form of the function $\phi^{0}(r)$ in terms of the quantum mass as

$\phi^{0}(r)=\sqrt{\frac{\Omega_{0}}{\mathcal{B}_{0}}} \sqrt{\frac{\mathcal{B}_{0}-\Omega_{0}-|M(r)|^{2}}{\Omega_{0}+|M(r)|^{2}}}$.

Given the functional form of $|M(r)|$ one can now easily determine the function $\phi^{0}(r){ }^{10}$

We also mention here that there are ways to make the quantum mass positive definite even in the classical background. For example, in [29], the authors had shown how to make $M$ positive definite by using a different interpretation of Bohmian mechanics and demanding that the mass $M$ should have the correct non relativistic limit. In this procedure, they obtained the following formula for the quantum mass $(M)$ for a particle of classical mass $m$

$M=m \exp \left[\frac{1}{m^{2}} \frac{\square \mathcal{R}}{\mathcal{R}}\right]$.

With this mass formula, one can obtain the correct non relativistic equation of motion of the particle as shown in [29]. The resulting theory has been studied extensively in the context of conformal transformations and curved spacetime (see [11] for a review). ${ }^{11}$ In this paper, we have already shown a way out of this problem by using the disformal transformation, nevertheless it is useful to make the theory free from any tachyonic solution from the start. We leave the problem of incorporating the mass of Eq. (67) in our transformation formulas of Eq. (55) for a future study.

\section{Conclusions}

In the well known approach of incorporating the quantum effects in spacetime geometry, conformally related spacetimes are used to establish the equivalence between the Bohmian motion of a particle on a classical background with force free classical motion on a quantum corrected background. In terms of this bimetric description of gravity, the Bohmian force can be interpreted as arising due to the description of the particle in the gravitational geometry rather than in the physical one, and quantum effects are incorporated in the conformal degrees of freedom [13]. This line of reasoning has been used to argue that the singularity problem of GR can be avoided in this approach [6]. The first part

\footnotetext{
${ }^{10}$ Of course for some $|M(r)|$ the function $\phi^{0}(r)$ determined in Eq. (66) may not be real every where. In that case one has to take an ansatz for the vector field different from the one given here.

11 This approach was recently used in [23] to derive a quantum version of the Friedmann equations.
}

of this paper is devoted to the discussion of various aspects of this approach and we have also pointed out some of the problematic issues of this approach.

In the second part, to overcome these limitations of the conformal transformation we have considered transformations of the metric more general than the conformal once, namely the disformal transformation proposed by Bekenstein in the context of bi-metric gravity. The conformal transformation is equivalent to a uniform coordinate dependent scaling in every spacetime direction. On the other hand, in a disformal transformation of the form given in Eq. (31), we not only perform a uniform scaling in every direction (conformal part), we also scale a particular direction (chosen by the normal vector $\phi_{\mu}$ ) differently from other directions. This fact is more transparent from the so called pure disformal transformation, where we only scale the direction chosen by the velocity vector $v_{\mu}$, and the other directions (perpendicular to $v_{\mu}$ in an orthonormal coordinate frame) do not scale at all i.e., the conformal factor is just a constant.

We have shown how one can incorporate the quantum nature of a particle (both massive and massless) in the disformal degrees of freedom of the physical geometry. This implies that the quantum effects can be viewed as a disformal transformation between the gravitational and the physical geometry, i.e., if we consider the motion of quantum particle in the gravitational geometry, due to the presence of the quantum potential this is equivalent to the classical free fall motion in a disformally transformed spacetime where the transformation is done along the direction chosen by the the particle 4 velocity. The quantum force can thus thought to be arisen in the gravitational geometry only because we had chosen the "wrong" frame for the analysis. Had we started from the disformally related physical geometry itself the quantum force would have never arise and the motion would have been classical. Most importantly, by using disformal transformation we have shown here that one can solve the usual problems in the conformal version, such as wrong continuity equation and the problem of definiteness of mass.

Before concluding we mention here another future application of the formalism constructed here. We notice that the second factor in the transformation relation of expansion scalar derived in Eq. (26) indicates that it is not a conformally invariant quantity. Similarly it is easy to show, taking symmetric and antisymmetric part of Eq. (25) respectively that, the shear and rotation tensors are also not conformally invariant. Applying directional derivative to Eq. (26) we see that Raychaudhuri equation is also not conformally invariant

$$
\frac{d \tilde{\theta}}{d \tilde{\tau}}=\Omega^{-2}\left[\frac{d \theta}{d \tau}-\Omega^{-1}\left(\theta+3 \frac{d \ln \Omega}{d \tau}\right) \frac{d \Omega}{d \tau}+3 \frac{d^{2} \ln \Omega}{d \tau^{2}}\right] .
$$


In GR, such quantities are problematic because for a particle trajectory these are the observable quantities and appear in the Raychudhuri equation in their scalar form, so these should be conformally invariant. The root of this problem lies in the transformation relation of Christoffel symbols Eq. (24) and the fact that in GR Christoffel connections are symmetric in the lower indices. This makes geodesic equation to changes differently in conformally related frames. This problem has recently been addressed in [30], where the authors have shown how to make the geodesic equation invariant under a conformal transformation in the framework of Einstein-Cartan gravity, where the presence of non zero torsion makes this possible. It will be interesting to see how such modifications can affect the quantum motion of a particle in curved spacetime along the lines discussed in this paper.

Data Availability Statement This manuscript has no associated data or the data will not be deposited. [Authors' comment: This work being theoretical in nature did not use any data. Hence no data is associated with this paper.]

Open Access This article is licensed under a Creative Commons Attribution 4.0 International License, which permits use, sharing, adaptation, distribution and reproduction in any medium or format, as long as you give appropriate credit to the original author(s) and the source, provide a link to the Creative Commons licence, and indicate if changes were made. The images or other third party material in this article are included in the article's Creative Commons licence, unless indicated otherwise in a credit line to the material. If material is not included in the article's Creative Commons licence and your intended use is not permitted by statutory regulation or exceeds the permitted use, you will need to obtain permission directly from the copyright holder. To view a copy of this licence, visit http://creativecomm ons.org/licenses/by/4.0/.

Funded by $\mathrm{SCOAP}^{3}$.

\section{References}

1. J.D. Bekenstein, Phys. Rev. D 48, 3641 (1993)

2. D. Bohm, B.J. Hiley, The Undivided Universe (Routledge, New York, 1993)
3. P. Holland, The Quantum Theory of Motion (Cambridge University Press, Cambridge, 1993)

4. D. Durr, S. Teufel, Bohmian Mechanics (Springer, New York, 2009)

5. F. Shojai, M. Golshani, Int. J. Mod. Phys. A 13(4), 677-693 (1998)

6. S. Das, Phys. Rev. D 89, 084068 (2014)

7. A.F. Ali, S. Das, Phys. Lett. B 741, 276-279 (2015)

8. A.F. Ali, M.M. Khalil, Nuc. Phys. B 909, 173 (2016)

9. F. Rahmani, M. Golshani, Int. J. Mod. Phys. A 33(3), 1850027 (2018)

10. B. Koch, arXiv:0810.2786

11. R. Carroll, Fluctuations, Information, Gravity and the Quantum Potential (Springer, New York, 2006)

12. I. Licata, D. Fiscaletti, Quantum Potential: Physics, Geometry and Algebra (Springer, New York, 2014)

13. A. Shojai, F. Shojai. arXiv:gr-qc/0404102

14. S. Jalalzadeh, A.J.S. Capistrano, Mod. Phys. Lett. A 34(33), 1950270 (2019)

15. T. Takabayasi, Prog. Theor. Phys. 9, 187 (1953)

16. T. Chiba, F. Chibana, M. Yamaguchi, JCAP 2006, 003 (2020)

17. S. Carroll, Spacetime and Geometry-An Introduction to General Relativity (Pearson, London, 2003)

18. Y. Fujii, K. Maeda, The Scalar-Tensor Theory of Gravitation (Cambridge, 2003)

19. I. Quiros, Int. J. Mod. Phys. D 28(07), 1930012 (2019)

20. M.P. Dabrowski, J. Garecki, D.B. Blascheke, Ann. Phys. (Berlin) 18(1), 13-32 (2009)

21. R. Wald, General Relativity (Chicago Press, 1984)

22. A.K. Kembhavi., MNRAS 185, 807 (1978)

23. G. Gregori, B. Reville, B. Larder, Astrophys. J. 886, 50 (2019)

24. E. Poisson, A Relativist's Toolkit (Cambridge University Press, Cambridge, 2004)

25. D. Kothawala, Gen. Relativ. Gravit. 46, 1836 (2014)

26. G. Domènech, A. Naruko, M. Sasaki, JCAP 1510, 067 (2015)

27. D. Bettoni, S. Liberati, Phys. Rev. D 88, 084020 (2013)

28. M. Zumalacárregui, J. García-Bellido, Phys. Rev. D 89, 064046 (2014)

29. A. Shojai, F. Shojai, Phys. Scr. 64, 413 (2001)

30. S. Lucat, T. Prokopec, Class. Quantum Gravity 33, 245002 (2016) 